\title{
In-situ Study of Coarsening Mechanisms of Supported Metal Particles in Reducing Gas
}

\author{
S.Y. Zhang ${ }^{1}$, Matteo Cargnello², Christopher B. Murray ${ }^{3}$, George W. Graham ${ }^{1}$, and Xiaoqing Pan ${ }^{4}$
}

${ }^{1}$ Dept of Materials Science and Engineering, University of Michigan, Ann Arbor, MI 48109, USA

${ }^{2}$ Dept of Chemical Engineering, Stanford University, Stanford, CA 94305, USA

${ }^{3}$ Dept of Chemistry and Department of Materials Science and Engineering, University of Pennsylvania, Philadelphia, PA 19104, USA

${ }^{4}$ Dept of Chemical Engineering and Materials Science and Department of Physics and Astronomy, University of California - Irvine, Irvine, CA 92697, USA

The ability to monitor dynamic material transformation processes in-situ is crucial for understanding structure-property relationships in nano-engineered materials. In the past decade, most of the in-situ gas reaction results were acquired with a dedicated environmental transmission electron microscope (ETEM) where the pressure in the sample area is typically around 10 Torr [1]. To make the in-situ condition more realistic, especially in terms of gas pressure, a MEMS-based closed cell technique has been developed recently [2]. Here we report results utilizing this breakthrough technique to study the coarsening mechanisms of supported metal particles within a conventional spherical aberration-corrected TEM at elevated temperature (above $500{ }^{\circ} \mathrm{C}$ ) and atmospheric pressure in real time, which provide important knowledge for designing and preparing more active and stable heterogeneous catalysts for specific reactions.

The gradual coarsening of metal nanoparticles in supported metal catalysts with time-in-use is one of the most widely recognized modes of catalyst deactivation. In general, particle coarsening occurs via a combination of two thermally activated processes, Ostwald ripening (OR), where larger particles grow at the expense of smaller ones through single-atom release and capture due to the size-dependent difference in surface energy, and particle migration/coalescence (PMC), which involves random motion of entire particles, leading to particle collisions and combination when they are in close enough proximity.

Mono-dispersed Pt nanoparticles, either $2.2 \pm 0.2 \mathrm{~nm}$ or $4.8 \pm 0.2 \mathrm{~nm}$ in diameter, were prepared as described previously [3]. Catalyst samples containing each, as well as a mixture of both, were subsequently prepared by wet impregnation of Sasol TH100-150 alumina. In-situ observation was carried out with a JEOL 3100 in combination with the Protochips Atmosphere ${ }^{\mathrm{TM}}$ system, which consists of a MEMS-based closed cell (two SiN windows, each 30-50 nm in thickness, with a $5 \mu \mathrm{m}$ gap in between), a heating holder, and a gas delivery manifold. The sample was heated under 760 Torr of $5 \% \mathrm{H}_{2} / \mathrm{N}_{2}$ at $600{ }^{\circ} \mathrm{C}, 700{ }^{\circ} \mathrm{C}$ and $800{ }^{\circ} \mathrm{C}$, each for $5 \mathrm{~h}$, in succession. The use of size-selected samples allows us to cleanly separate the two processes and study the corresponding mechanisms individually. In the sample containing both $2.2 \mathrm{~nm}$ and $4.8 \mathrm{~nm}$ particles, the dominant mechanism is OR, indicated by the gradual disappearance of the smallest particles in Figure 1. However, OR was successfully 
suppressed in both of the single-sized samples. Due to the higher mobility of the smaller particles, PMC results in a relatively small change in the particle size distribution of the sample containing the $2.2 \mathrm{~nm}$ particles only, as shown in Figure 2, while in the sample containing only $4.8 \mathrm{~nm}$ particles, essentially no change was observed after the total $15 \mathrm{~h}$ sintering experiment.

$E x$-situ observations revealed that beam-induced effects, such as surface disorder, particle rotation, and coalescence are pronounced for metal particles smaller than 5-6 nm with long exposure and high electron dose. Examples are shown in sequential images in Figure 3 and Figure 4. To avoid such artifacts, the beam valve needs to be closed between image acquisitions. The images used for particle size distribution measurements here were all taken under relatively low magnification, as a further precaution.

References

[1] DeLaRiva, A.T. et. al., J. Catal. 308 (2013) 291.

[2] Allard, L.F. et al., Microsc. Microanal. 18 (2012) 656.

[3] Cargnello, M., et al., Science 341 (2013) 771.

[4] The authors gratefully acknowledge funding from Ford Motor Company under a University Research Proposal grant and the National Science Foundation under grants DMR-0723032 and CBET-115940.
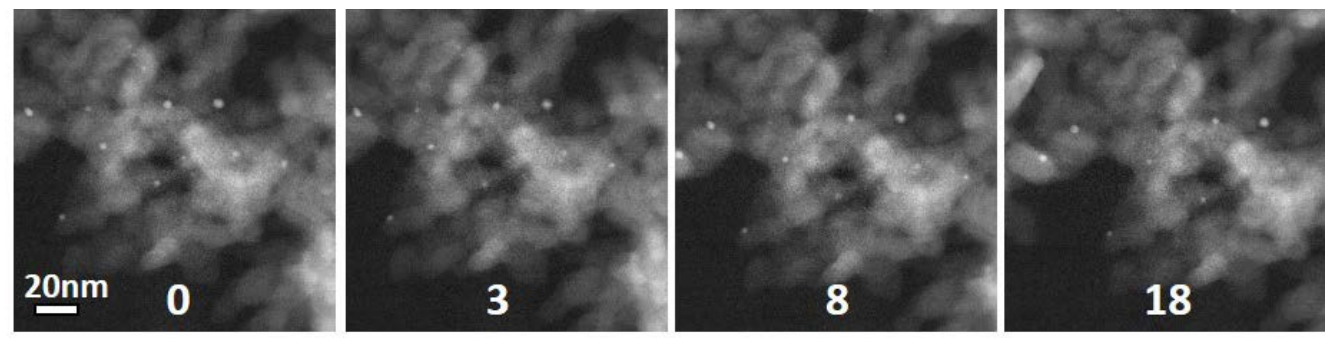

Figure 1. Sequential images showing the gradual disappearance of the smallest particles in the mixed sized sample at $800{ }^{\circ} \mathrm{C}$. Elapsed time, in minutes, is indicated on the bottom center of each image.
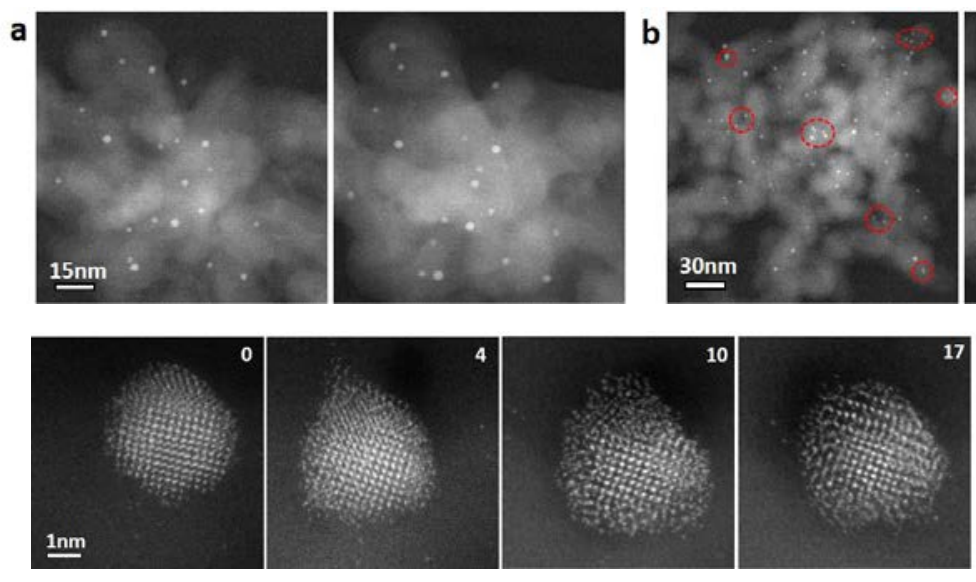

Figure 3. Sequential images showing beam-induced surface disorder. Elapsed time, in minutes, is indicated on the top right corner of each image.

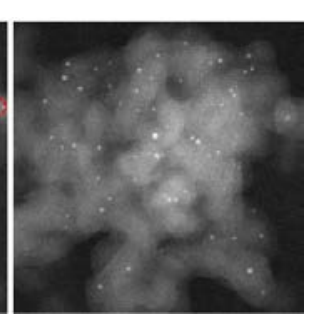

Figure 2. Comparison of low (a) and high (b) density areas before and after heating at $800{ }^{\circ} \mathrm{C}$ in the sample containing only the $2.2 \mathrm{~nm} \mathrm{Pt}$ particles.

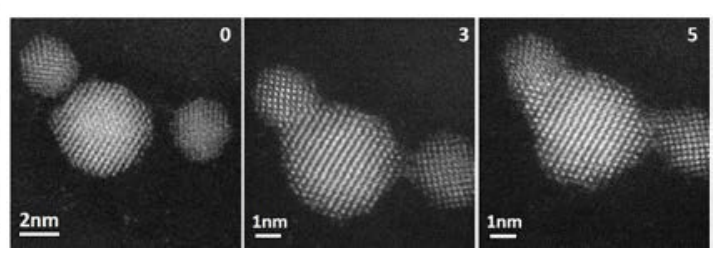

Figure 4. Sequential images showing beam-induced coalescence. Elapsed time, in minutes, is indicated on the top right corner of each image. 